\title{
Dynamics of the Oxygen Molecules Scattered from Graphite (0001) Surface and Comparison with Experimental Data
}

\author{
Víctor Morón a , Ludovic Martin-Gondre ${ }^{b, c}$, Pablo Gamallo ${ }^{a^{*}}$, and Ramón Sayós ${ }^{\text {a }}$ \\ ${ }^{a}$ Departament de Química Física and Institut de Química Teòrica i Computacional, Univ. Barcelona, \\ C/ Martí i Franquès 1, 08028 Barcelona, Spain \\ ${ }^{b}$ Donostia International Physics Center (DIPC), P. Manuel de Lardizabal 4, 20018 San Sebastián, Spain \\ ${ }^{c}$ Centro de Física de Materiales (CFM), Centro Mixto CSIC-UPV/EHU, P. Manuel de Lardizabal 5, \\ 20018 San Sebastián, Spain
}

\begin{abstract}
A quasiclassical trajectory dynamics study of molecular oxygen colliding over a free of defects and clean graphite (0001) surface has been performed with a recently published density functional theory based flexible periodic London-Eyring-Polanyi-Sato potential energy surface (PES). Although the PES was mainly constructed for describing accurately the recombination of atomic oxygen over an Opreadsorbed surface, here we show that this PES is also reliable to study the scattering of $\mathrm{O}_{2}$ over graphite surface. Thus, several initial conditions have been explored: collision energies $\left(0.2 \leq \mathrm{E}_{\mathrm{col}} \leq 1.2\right.$ $\mathrm{eV})$, incident angles $\left(\theta_{\mathrm{v}}=0^{\circ}, 45^{\circ}\right)$, surface temperatures $\left(100 \leq \mathrm{T}_{\text {surf }} \leq 900 \mathrm{~K}\right)$ and some rovibrational $\mathrm{O}_{2}$ levels $(\mathrm{v}=0,1,2$ and $\mathrm{j}=1,17,25)$. The calculated polar scattering angular distributions are in good agreement with the experimental ones in a wide range of explored conditions. Moreover, the comparison with hyperthermal experimental data, which was unclear in a previous work, has been finally clarified. The effect of $\mathrm{O}_{2}(\mathrm{v}, \mathrm{j})$ internal state on the scattering is very small.
\end{abstract}

Keywords: Quasiclassical trajectories, FPLEPS, oxygen scattering, $\mathrm{O}_{2}+$ graphite, potential energy surface, DFT calculations.

Tables: 1 Figures: 6

*Corresponding author: gamallo@ub.edu

Version: August 3rd 2012 


\section{Introduction}

Satellites and other orbiting vehicles in low Earth orbits (i.e., at 160 - $2000 \mathrm{Km}$ above Earth) must travel at velocities around $7.5 \mathrm{~km} / \mathrm{s}$ in order to avoid collapsing on Earth's surface due to gravity. At these high velocities the shielding of the vehicle receives a great amount of highly energetic collisions (i.e., about $\left.4.5 \pm 1.0 \mathrm{eV}^{1}\right)$ of the main present species $\left(\mathrm{O} / \mathrm{O}_{2}\right)$. These collisions can produce several heterogeneous processes (etching, adsorption, reflection, recombination, oxidation) and also a flux of heat to the surface of the vehicle. It is worth for space industry to model this kind of elementary processes in order to improve the behaviour of the thermal protection systems of space vehicles at these extreme conditions. Among these processes, the oxidation reaction between $\mathrm{O}_{2}$ and several carbonmade materials such as graphite, graphene and single-wall carbon nanotubes is known to play an important role not only during these reentries but also in many other fields (e.g., heterogeneous oxidation catalysis, solubility and functionalization of single-wall carbon nanotubes). The oxidation of these materials alters their physicochemical properties such as the wettability, adsorption (sticking) of the surface and use to be a drawback for their technological application. It is known that the molecular chemisorption and the dissociative adsorption of $\mathrm{O}_{2}$ molecule over carbon materials depend on the availability of reactive sites (i.e., graphitic edge planes or defects ${ }^{2}$ ) and also on the surface temperature. It is important to note that graphite with defect-free basal planes undergoes thermal oxidation at extremely high temperatures whereas at low-temperatures exposure leads to only weakly physisorbed $\mathrm{O}_{2}$. According to this, a pristine (i.e., free of defects) graphite surface would be the more appropriate and desirable for acting as a thermal protection system since the number of surface reactions would be minimized and also the heat transfer to the surface.

Recently, some experiments ${ }^{3,4}$ reported the polar scattering angular distributions resulting from a highly collimated and nearly monoenergetic incident beam of $\mathrm{O}_{2}$ scattered from the surface of highly 
oriented pyrolitic graphite. An important characteristic of the experimental setup is the use of a fixed source-detector geometry in which the angle between the incident beam and the detector direction was held at an angle of $90^{\circ}$. These experiments were carried out at the particular incident energy within the range $0.291 \mathrm{eV} \leq \mathrm{E}_{\mathrm{col}} \leq 0.614 \mathrm{eV}$ and also for a surface temperature in the interval $\left(150 \mathrm{~K} \leq \mathrm{T}_{\text {surf }} \leq 500\right.$ K). For each collision energy and surface temperature the angular distribution intensities were reported as a function of final polar scattering angle $\left(\theta_{v}{ }^{\prime}\right)$ relative to the surface normal. Moreover, some experiments $5,6,7$ were also performed with hyperthermal beams of $\mathrm{O}\left({ }^{3} \mathrm{P}\right)$ atoms with some extent of $\mathrm{O}_{2}\left(\mathrm{X}^{3} \Sigma_{\mathrm{g}}{ }^{-}\right)$that impinge over highly oriented graphite. In a previous work ${ }^{10}$, we showed that the quasiclassical trajectory (QCT) polar scattering angular distributions, $\mathrm{I}\left(\theta_{\mathrm{v}}{ }^{\prime}\right)$, of the $\mathrm{O}_{2}$ molecules formed by the Eley-Rideal reaction presented a peak centred at $\theta_{\mathrm{v}}{ }^{\prime}=\sim 40^{\circ}$ much lower than the experimental $\mathrm{O}_{2}$ distribution, peaked at $\theta_{\mathrm{v}}{ }^{\prime}=\sim 60^{\circ}{ }^{5}$. This discrepancy is understood since in the experiment, a mixture of $\mathrm{O}_{2} / \mathrm{O}$ forms the initial beam, so the higher contribution to the $\mathrm{O}_{2}$ polar scattering angle distribution should come from the $\mathrm{O}_{2}$ molecules directly scattered by the surface. Thus, in order to elucidate this point we have completed our previous Eley-Rideal QCT study ${ }^{10}$ with the addition of a new $\mathrm{O}_{2}$ QCT scattering analysis for the same initial experimental conditions.

This paper presents a QCT dynamics study of $\mathrm{O}_{2}$ molecules colliding with a pristine graphite (0001) surface. Our recent Flexible Periodic London-Eyring-Polanyi-Sato (FPLEPS) potential energy surface (PES) based on density functional theory (DFT) data has been used. This PES was used previously for describing the O collisions with a clean ${ }^{8,9}$ and with an O-preadsorbed ${ }^{10}$ graphite (0001) surface, obtaining a good agreement with experimental data. As above mentioned, thermal oxidation on free of defects graphite surfaces only occur at extremely high temperatures, so the FPLEPS surface (for a graphite surface without any defects) must be accurate enough for describing the main process (i.e., molecular scattering) occurring during the collision. The use of an analytical expression for the PES 
allows obtaining easily and efficiently the potential energy and its first order derivatives for performing fast dynamics calculations. Some extra DFT calculations were also performed in order to know the accuracy of the analytical PES in describing the $\mathrm{O}_{2}$ interaction with the graphite surface.

This paper is organized as follows: Section 2 provides a brief description about DFT and QCT calculations; Section 3 describes the FPLEPS vs. DFT comparison of the $\mathrm{O}_{2}$ scattering (i.e., reflection) over graphite and Section 4 presents the main dynamical results and discussion, including the comparison with experimental data. Finally, Section 5 gives the summary and conclusions.

\section{Theoretical methods}

\section{B. DFT calculations}

Periodic density functional theory calculations have been performed using the Vienna ab initio simulation package (VASP) ${ }^{11,12,13,14}$. All the details about the methodology used (e.g., GGA/RPBE functional, energy cut off, slab model, ...) were discussed deeply in previous works ${ }^{8,9,10}$ devoted to atomic oxygen interaction with both a clean and an O-preadsorbed graphite surfaces, and are omitted here. New DFT calculations have been performed in order to check the accuracy of the analytical FPLEPS PES in describing the $\mathrm{O}_{2} /$ graphite interaction. According to the previous study ${ }^{9}$, it is important to remember that the molecular channel does not present any particular feature; it is essentially repulsive close to the surface and a high endothermicity $(5.22 \mathrm{eV})$ prevents the molecular dissociation at the conditions studied here. Thus, the DFT data used to construct the analytical surface were focused mainly on the Eley-Rideal entrance channel. To check the proper description of the interaction of the incoming molecule with the surface, some additional configurations at several particular $\phi$ angles (Figure 1) were calculated at DFT level and compared with the data obtained from 
the FPLEPS PES, with a good agreement as described below in Section III.

\section{B. QCT calculations}

A dynamical study involving the interaction of molecular oxygen with graphite surface was performed by means of the QCT method ${ }^{15}$. Several initial conditions were sampled in order to investigate state-specific and hyperthermal processes. State-specific calculations $\left(\mathrm{v}, \mathrm{j}, \mathrm{E}_{\mathrm{col}}, \mathrm{T}_{\mathrm{surf}}, \theta_{\mathrm{v}}\right)$ were carried out for fixed initial collision energies in the range of $0.2 \mathrm{eV} \leq \mathrm{E}_{\mathrm{col}} \leq 1.2 \mathrm{eV}$ for a given surface temperature $\mathrm{T}_{\text {surf }}$ within the $100-900 \mathrm{~K}$ interval and several vibrorotational states $(\mathrm{v}=0,1,2$ and $\mathrm{j}=1,17,25)$. The incoming velocity angle $\left(\theta_{\mathrm{v}}\right.$ in Figure 1$)$ is defined with respect to the negative $\mathrm{Z}$ axis (i.e., $0^{\circ}$ for normal incidence) and its projection onto the $\mathrm{X}-\mathrm{Y}$ plane (i.e., the azimuthal $\phi_{\mathrm{v}}$ angle) was uniformly sampled within the $0^{\circ}-360^{\circ}$ interval since highly oriented graphite surface consists of many orientations within the topmost layer, being the measurements an average of all azimuthal directions ${ }^{16}$.

The initial collision angles selected for the present study were fixed values at $\theta_{\mathrm{v}}=0^{\circ}$ and $45^{\circ}$, and also a random sampling within the $10^{\circ} \leq \theta_{\mathrm{v}} \leq 70^{\circ}$ range. Initial molecular orientation angles $(\theta, \phi$ in Figure 1) were sampled by the standard Monte Carlo method within the intervals $0^{\circ} \leq \theta \leq 180^{\circ}$ and $0^{\circ}$ $\leq \phi \leq 360^{\circ}$, respectively. The initial $r$ internuclear distance was chosen between the corresponding inner (r-) and outer $\left(r_{+}\right)$turning points of each $\mathrm{O}_{2}(\mathrm{v}, \mathrm{j})$ internal state. Only odd j initial levels were chosen for $\mathrm{O}_{2}\left(\mathrm{X}^{3} \Sigma^{-} \mathrm{g}\right)$ molecule because even rotational levels do not exist due to the nuclear spin of $\mathrm{O}^{16}$ is zero ${ }^{17}$. Initial position $\left(\mathrm{X}_{\mathrm{cm}}, \mathrm{Y}_{\mathrm{cm}}\right)$ of the molecular centre of mass was randomly selected inside the $(1 \times 1)$ unit cell while $\mathrm{Z}_{\mathrm{cm}}$ was set at $7.0 \AA$, where the interaction with surface is negligible.

A Generalized Langevin Oscillator (GLO) model ${ }^{18,19,20}$ was used in order to account for the energy 
exchange between the molecule and the solid surface, which was considered as a rigid slab (i.e., 6D PES). This model includes the surface and ghost particle motions into the Hamilton equations and uses also the same optimum parameters, which were previously derived and tested for atomic oxygen impinging on both clean ${ }^{9}$ and O-preadsorbed graphite surfaces ${ }^{10}$. The oscillator surface frequencies used were $\omega_{\mathrm{i}, \mathrm{x}}=\omega_{\mathrm{i}, \mathrm{y}}=10^{-3}$ and $\omega_{\mathrm{i}, \mathrm{Z}}=3.4 \times 10^{-4}$ au for $i=1,2,3$, with an effective mass of $60 \mathrm{amu}$ and with friction constants $\gamma_{\mathrm{g}, \mathrm{x}}=\gamma_{\mathrm{g}, \mathrm{y}}=\gamma_{\mathrm{g}, \mathrm{z}}=4.0 \times 10^{-4} \mathrm{au}$. The need for an effective mass much larger than that of a single carbon atom has been documented previously, and points out a collective scattering from a large number of surface carbon atoms ${ }^{3,4}$.

The qctsurf code developed in our group was used to calculate the trajectories integrating the Hamilton equations of the system (included the GLO) using the Beeman algorithm. The time step used was $5 \times 10^{-17} \mathrm{~s}$, which ensures a total energy conservation along the trajectories lower than $1 \times 10^{-4} \mathrm{eV}$ in absence of the thermal bath. The total energy is not constant due to the thermal bath dissipation effect. Total integration time was set to a maximum value of $2.5 \mathrm{ps}$, that allows a correct classification of the trajectories as reflected, since no other channels were observed. Anyway, the adsorption classification was set for $\mathrm{Z}_{\mathrm{cm}}$ values lower than $2.2 \AA$ and for a number of total molecular rebounds with the surface larger than 8 , as was also set previously ${ }^{9,10}$ for atomic oxygen impinging a clean or an O-preadsorbed graphite surface. On the other hand, $\mathrm{O}_{2}$ was considered reflected if $\mathrm{Z}_{\mathrm{cm}}$ was higher than $7.1 \AA$ and the direction of the velocity vector pointed to the vacuum.

The number of total trajectories $\left(\mathrm{N}_{\mathrm{T}}\right)$ calculated for each state-specific condition was around 20000 . However, the number of trajectories carried out for comparing with experimental results was increased for some conditions. Thus, for simulating the hyperthermal experimental conditions of Paci et al. ${ }^{5}$ a set of 60000 trajectories were performed while for simulating the experimental setup of Oh et al. ${ }^{3}$ batches of 400000 trajectories were run for each $\left(\mathrm{v}, \mathrm{j}, \mathrm{E}_{\mathrm{col}}, \mathrm{T}_{\text {surf }}\right)$ condition. 


\section{Analytical FPLEPS potential energy surface}

The complete expression used for describing the $6 \mathrm{D}$ potential $\mathrm{V}\left(\mathbf{R}_{\mathrm{A}}, \mathbf{R}_{\mathrm{B}}\right)$ of the two atoms $(\mathrm{A}, \mathrm{B})$ interacting with a rigid graphite surface (Figure 1) was defined as 9,10 :

$$
\mathrm{V}\left(\mathrm{R}_{\mathrm{A}}, \mathrm{R}_{\mathrm{B}}\right)=\mathrm{U}_{\mathrm{As}}+\mathrm{U}_{\mathrm{Bs}}+\mathrm{U}_{\mathrm{AB}}-\sqrt{\mathrm{Q}_{\mathrm{AB}}+\left(\mathrm{Q}_{\mathrm{As}}+\mathrm{Q}_{\mathrm{Bs}}\right)^{2}-\mathrm{Q}_{\mathrm{AB}} \cdot\left(\mathrm{Q}_{\mathrm{As}}+\mathrm{Q}_{\mathrm{Bs}}\right)}+\mathrm{G}_{1}+\mathrm{G}_{2}
$$

where the positions of the two atoms $A$ and $B$ are given by the vectors $\mathbf{R}_{A}\left(X_{A}, Y_{A}, Z_{A}\right)$ and $\mathbf{R}_{\mathrm{B}}\left(\mathrm{X}_{\mathrm{B}}, \mathrm{Y}_{\mathrm{B}}, \mathrm{Z}_{\mathrm{B}}\right)$, respectively. $\mathrm{G}_{1}$ and $\mathrm{G}_{2}$ are two Gaussian functions introduced to improve the description of the full potential in the entrance valley. The terms $U_{i}$ and $Q_{i}$ represent respectively the Coulomb and exchange integrals for two-body systems and are associated with the atom-surface interaction $(\mathrm{i}=\mathrm{As}, \mathrm{Bs})$ and with the molecular interaction $(\mathrm{i}=\mathrm{AB})$. The full description of the expressions and the optimal PES parameters for $\mathrm{O}_{2}$ /graphite system were reported previously ${ }^{10}$.

As commented in previous Section II.A, the analytical FPLEPS surface was checked for the $\mathrm{O}_{2(\mathrm{~g})}$ scattering channel by comparing with additional DFT data. Thus, a total of 423 molecular DFT configurations were calculated for a parallel approach of the $\mathrm{O}_{2}$ molecule $\left(\theta=90^{\circ}\right)$ with two $\phi$ angles $\left(0^{\circ}\right.$ and $60^{\circ}$, Figure 1$)$ over a $(3 \times 3)$ unit cell, with the molecular centre of mass fixed over the bridge site (B1) and with perpendicular velocity to the surface $\left(\theta_{\mathrm{v}}=0^{\circ}\right)$ at several $\mathrm{Z}_{\mathrm{cm}}$ values between $1.3 \AA$ and $5.0 \AA$ and for internuclear distances in the range $1.15 \AA \leq r \leq 4.0 \AA$. The DFT contour plots (Figure 2) show that no molecular adsorption is present and that only molecular dissociative adsorption onto no consecutive bridge sites is observed. Thus, for $\phi=0^{\circ}$, the distance between the two bridge sites where the atomic adsorption occurs is $4.936 \AA$ while for $\phi=60^{\circ}$ the distance is shorter $(2.468 \AA)$. Nevertheless, the process is highly endothermic (5.22 eV). The comparison with the FPLEPS contour plots is also shown in Figure 2, where the agreement is quite good without the necessity of including 
additional DFT points in the fitting procedure. The root mean square deviation (RMSD) for energy values lower than $1.0 \mathrm{eV}$ ( 240 points) is $0.3 \mathrm{eV}$. This value is not too small but the reason is that $\mathrm{O}_{2}$ dissociation energy value used in the FPLEPS PES is the one obtained from more accurate coupledcluster calculations ${ }^{21}$ instead of the less accurate DFT ones, hence giving to the FPLEPS PES a good description for both asymptotes. The RMSD value without considering the $\mathrm{O}_{2}$ entrance valley diminishes until $0.12 \mathrm{eV}$.

\section{QCT dynamical results}

\section{A. State-specific initial conditions}

When $\mathrm{O}_{2}$ impinges the graphite surface three principal channels are available: molecular adsorption, reflection and dissociation, which can produce either gas or adsorbed species: $\mathrm{O}_{(\mathrm{g})}+\mathrm{O}_{(\mathrm{g})}, \mathrm{O}_{(\mathrm{ad})}+\mathrm{O}_{(\mathrm{g})}$, $\mathrm{O}_{(\mathrm{g})}+\mathrm{O}_{(\mathrm{ad})}$. From all of these possible processes only molecular reflection is observed at all studied conditions. The effect of the initial collision energy (Figure 3) on the polar scattering angular distributions $\left(I\left(\theta_{v}{ }^{\prime}\right)\right.$, where $\theta_{v}{ }^{\prime}$ is defined as the angle between the final centre of mass velocity and the positive Z-axis) of the molecules reflected has been investigated for a selected vibrorotational initial state of $\mathrm{O}_{2}$ (e.g., $\mathrm{v}=0$ and $\mathrm{j}=1$ ). Thus, for normal incidence, the molecules are scattered at low angles $\left(\theta_{\mathrm{v}}{ }^{\prime}=10^{\circ}-20^{\circ}\right)$ while for off normal incidence $\left(\theta_{\mathrm{v}}=45^{\circ}\right)$ the scattering angle distributions present a peak at around the specular value $\left(\theta_{\mathrm{v}}{ }^{\prime}=45^{\circ}\right)$ for low collision energy $\left(\mathrm{E}_{\mathrm{col}}=0.2 \mathrm{eV}\right)$ with and without including surface temperature. The peaks become superspecular for higher collision energies when the surface temperature is included (i.e., $\theta_{\mathrm{v}}{ }^{\prime} \approx 60^{\circ}$ at $\mathrm{E}_{\mathrm{col}}=1.2 \mathrm{eV}$ and $\mathrm{T}_{\text {surf }}=100,900 \mathrm{~K}$ ). These results are similar to those obtained for the atomic reflection on a clean graphite surface (Figure 6 in Ref. 9) at low 
collision energy $\left(\mathrm{E}_{\mathrm{col}}=0.25 \mathrm{eV}\right)$. Nevertheless, at higher collision energies (i.e., $\mathrm{E}_{\mathrm{col}}=0.5$ and $1.3 \mathrm{eV}$ ) the atomic reflection drove to wider scattering distributions not observed here for the molecular case. The analysis of QCT results shows that the collision time for all the conditions explored is very short with respect to the total maximum time initially chosen for the integration (i.e., $2.5 \mathrm{ps}$ ). Specifically at normal incidence and without thermal bath the average collision time was 0.56 and $0.41 \mathrm{ps}$ at $\mathrm{E}_{\mathrm{col}}=0.2$ and $1.2 \mathrm{eV}$, respectively, while for off normal incidence the average times were slightly higher: 1.02 and $0.52 \mathrm{ps}$ for the same collision energies, respectively. When the thermal bath is included, the collision time is nearly doubled for all the conditions explored. This fact supports that the scattering of the molecule with the surface is dominated essentially by a single collision event.

The influence of the initial vibrational and rotational $\mathrm{O}_{2}$ excitation on the final polar scattering angular distribution has been also investigated for $\mathrm{O}_{2}(\mathrm{v}=0,1,2$ and $\mathrm{j}=1,17,25)$ showing a negligible effect.

Figure 4 shows the different types of energy exchanged (i.e., $\Delta E_{i}=E_{i}^{\prime}-E_{i}$ ) during the molecular collision ( $i$ corresponds to translational, internal and total energy) for three initial collision energies, $\mathrm{O}_{2}$ $(\mathrm{v}=0, \mathrm{j}=1)$ with normal incidence and at two surface temperatures. In general, collision energy of impinging molecules is lost during the interaction process (Figures $4 \mathrm{a}$ and $4 \mathrm{~b}$ ) although at higher temperatures some intensity appears at positive values (i.e., some energy release from the surface to the molecule, Figure 4b). A fraction of the initial collision energy is going to molecular rotational excitation (no vibrational), as can be seen from the positive values of the internal energy exchanged (Figures $4 \mathrm{c}$ and $4 \mathrm{~d}$ ). This increment is slightly higher when the surface temperature is augmented. The major part of the initial collision energy lost is transferred to the surface (Figures $4 \mathrm{e}$ and $4 \mathrm{f}$ ). Only at the highest $T_{\text {surf }}$ explored and for low collision energies $\left(E_{\mathrm{col}}=0.2\right.$ and $\left.0.5 \mathrm{eV}\right)$ some intensity is observed at positive values of total energy, showing some energy release from the surface to the 
molecule. In general, the total energy transferred to the surface increases with the augment of collision energy as could be expected.

The initial $\mathrm{O}_{2}$ vibrational state is conserved during the collision with the surface, whereas an important rotational excitation is observed. For example, for $\mathrm{E}_{\mathrm{col}}=0.2,0.5$ and $1.2 \mathrm{eV}$ and normal incidence without thermal bath and with $\mathrm{O}_{2}$ at $\mathrm{v}=0$ and $\mathrm{j}=1$ state, the reflected $\mathrm{O}_{2}$ molecules appear also in their ground vibrational state $\left(\mathrm{v}^{\prime}=0\right)$ but populating a great amount of rotational states (i.e., $\mathrm{j}^{\prime}>=16,25$ and 38 , respectively). When the surface temperature is considered, the same trend is observed although the final rotational excitation is lower. For an initial excited vibrorotational state (e.g., $\mathrm{O}_{2}$ at $\mathrm{v}=2$ and $\mathrm{j}=25$ ), normal incidence and $\mathrm{E}_{\mathrm{col}}=2.0 \mathrm{eV}$, the final scattered molecules also mainly appear at v' $=2$ state $(88.1 \%)$, although some of them are observed at lower or higher states: v' $=0(0.06 \%), v^{\prime}=1(7.46 \%), v^{\prime}=3(4.37 \%)$ and $v^{\prime}=4(0.01 \%)$.

Particular attention has been devoted in simulating the experimental conditions of Oh et al. ${ }^{3}$ For each pair of initial collision energy and surface temperature, the polar scattering angular distribution is reported, taken into account that the incident $\left(\theta_{\mathrm{v}}\right)$ and the final $\left(\theta_{\mathrm{v}}{ }^{\prime}\right)$ velocity angles, both relative here to the surface normal, must accomplish the relationship $\theta_{\mathrm{v}}+\theta_{\mathrm{v}}{ }^{\prime}=90^{\circ}$. This particular source-detector geometry setup makes that a great amount of QCT trajectories (i.e., 400000) must be integrated, since only those that verify the previous relationship $(\sim 1 \%)$ match the experimental conditions. Nevertheless, if no constraint is imposed between initial and final angles, for a particular incoming polar velocity angle (e.g., $\left.\theta_{\mathrm{v}}=45^{\circ}\right)$ the final scattering distribution achieved is very similar to those shown in Figures $3 \mathrm{~d}$ and $3 \mathrm{f}$, depending on the surface temperature considered. Calculations where run at each specific experimental surface temperature $\left(150 \mathrm{~K} \leq \mathrm{T}_{\text {surf }} \leq 500 \mathrm{~K}\right)$ and initial collision energy $\left(0.291 \mathrm{eV} \leq \mathrm{E}_{\mathrm{col}} \leq\right.$ $0.614 \mathrm{eV}$ ), choosing randomly the initial polar velocity angle within the range $10^{\circ} \leq \theta_{\mathrm{v}} \leq 70^{\circ}$. The angular tolerance in the final scattering angle $\left(\theta_{\mathrm{v}}{ }^{\prime}\right)$ was set to $1^{\circ}$. In the experimental conditions the 
vibrational temperature should be almost the same as the nozzle temperature (i.e., $300 \mathrm{~K}$ for $0.291 \mathrm{eV}$ and $700 \mathrm{~K}$ for $0.614 \mathrm{eV}^{16}$ ) and the rotational temperature is estimated to be very low after a free-jet expansion (i.e., $10 \mathrm{~K}$ for $0.291 \mathrm{eV}$ and $55 \mathrm{~K}$ for $0.614 \mathrm{eV}^{16}$ ). Therefore, we assumed the lowest vibrorotational $\mathrm{O}_{2}$ state (i.e., $\mathrm{v}=0, \mathrm{j}=1$ ) in QCT simulations.

Figure 5 shows the comparison between experimental ${ }^{3}$ and QCT polar scattering angular distributions of the reflected oxygen molecules at all the explored experimental conditions. The angular distributions consisted of single broad peaks with the most probable intensity occurring at an angle slightly larger than the specular position. Increasing the surface temperature, the maximum intensity decreases and the width increases. As Oh et al. ${ }^{3}$ suggested, this behaviour is indicative of a single scattering collision that can be described by classical mechanics where the collision involves short times, large energy transfers and exchange of large numbers of phonons or electronic excitations ${ }^{22}$ in the same manner that our QCT results state. An additional analysis of a sample of trajectories confirms a direct scattering mode for several collision energies and surface temperatures.

From these panels it is important to note that the shape of the QCT distributions are essentially coincident with experimental distributions although the QCT peaks are slightly shifted to lower angles mainly for $\mathrm{E}_{\mathrm{col}} \leq 0.5 \mathrm{eV}$. From Table $\mathrm{I}$ it is easy to see that the value of this shift at the maximum of the distributions oscillates within the interval $5.0^{\circ}-1.8^{\circ}$. The maximum deviation is obtained for low surface temperatures (i.e., $\mathrm{T}_{\text {surf }}=200-250 \mathrm{~K}$ ) at $\mathrm{E}_{\mathrm{col}}=0.291 \mathrm{eV}$, and the best agreement is reached for the highest collision energy (i.e., $\mathrm{E}_{\mathrm{col}}=0.614 \mathrm{eV}$ and $\mathrm{T}_{\text {surf }}=150 \mathrm{~K}$ ).

In spite of the QCT distribution are normalized to the maximum to enable an easy comparison with the experimental distributions, the unnormalized QCT distributions show also the same intensity trends as the experimental ones. Thus, the peak intensities increase when $\mathrm{E}_{\text {col }}$ does and also when $\mathrm{T}_{\text {surf }}$ decreases, in agreement with the experimental behaviour. 
Table I also presents the full width at half-maximum (fwhm) for each distribution. The agreement in fwhm values is quite good for almost all the cases studied and excellent for some particular cases. For example, at $\mathrm{T}_{\text {surf }}=150 \mathrm{~K}$ when $\mathrm{E}_{\mathrm{col}}$ increases, the fwhm value diminishes experimentally. This trend is in good agreement with QCT values although a slight increase is observed at $\mathrm{E}_{\mathrm{col}}=0.371 \mathrm{eV}$. The difference between QCT and experimental ${ }^{3} \mathrm{fwhm}$ values (hereafter $\Delta \mathrm{fwhm}$ ) is $-0.1^{\circ}, 1.5^{\circ}, 2.0^{\circ}, 2.5^{\circ}$ and $2.8^{\circ}$ for $0.291,0.371,0.456,0.534$ and $0.614 \mathrm{eV}$, respectively, at $\mathrm{T}_{\text {surf }}=150 \mathrm{~K}$. The same tendency is observed at $\mathrm{T}_{\text {surf }}=500 \mathrm{~K}$ for QCT and experimental fwhm values. In this case, when the initial $\mathrm{E}_{\text {col }}$ increases, the fwhm value decreases experimentally. The $\Delta$ fwhm values are $-2.1^{\circ},-0.5^{\circ}, 0.7^{\circ}, 1.0^{\circ}$ and $1.5^{\circ}$ for $0.291,0.371,0.456,0.534$ and $0.614 \mathrm{eV}$, respectively, at $\mathrm{T}_{\text {surf }}=500 \mathrm{~K}$.

The application of a hard cube model (HCM with an effective mass of $108 \mathrm{amu}$ ) fitting the experimental peak positions allow to explain the aforementioned experimental trends ${ }^{3}$ although with less accuracy than the present QCT results, which are only based on first principles. The introduction of the internal modes of $\mathrm{O}_{2}$ on the scattering through a smooth surface model ${ }^{3}$ or by using classical rigid molecular scattering calculations ${ }^{4}$ (both with an effective mass of $129.6 \mathrm{amu}$ ) improve the agreement with the experiment. However, the authors conclude that the small differences still observed between the theoretical and experimental distributions could be originated by other possible mechanisms caused by the presence of surface defects ${ }^{4}$, neither included in present QCT calculations.

Oh et al. ${ }^{3,4}$ stated by using a HCM that the translational energy of the molecules should be reduced by the collision with the graphite surface, in a similar manner as QCT results show (Figures 4a and 4b). In addition, they observed that the collision energy loss becomes smaller at higher surface temperatures. The amount of $\mathrm{E}_{\mathrm{col}}$ loss has been also calculated from QCT results at the peaks of the polar scattering angular distributions (Figure 5). Results are listed in Table I together with the theoretical results obtained by means of HCM results ${ }^{3,4}$, showing a quite good agreement. In the HCM 
model the tangential component of momentum of the colliding species is assumed as conserved during the single collision event with the surface. We have checked through the QCT calculations the validity of this hypothesis. Thus, for instance for $T_{\text {surf }}=150 \mathrm{~K}$ and two collision energies $(0.291$ and 0.614 $\mathrm{eV})$, the distribution of $\Delta \mathrm{E}_{\mathrm{col}, \|}=\mathrm{E}_{\mathrm{col}, \|}^{\prime}-\mathrm{E}_{\mathrm{col}, \|}$, for the trajectories corresponding at the peak of the polar scattering angular distributions (Figures $5 \mathrm{a}$ and $5 \mathrm{i}$ ), presents a curve with a maximum around zero in both cases, although slightly asymmetric and shifted to the negative values, confirming a reasonable agreement with this approximation. When we take into account not only the final trajectories that fulfils the experimental $\theta_{\mathrm{v}}+\theta_{\mathrm{v}}{ }^{\prime}=90^{\circ}$ restriction but also the other ones, this approximation becomes worse, indicating a major loss of the initial parallel collision energy. The analysis of the perpendicular

collision energy changes $\left(\Delta \mathrm{E}_{\mathrm{col}, \perp}=\mathrm{E}_{\mathrm{col}, \perp}^{\prime}-\mathrm{E}_{\mathrm{col}, \perp}\right)$ shows clearly that the loss of the initial collision energy is mainly due to its perpendicular component. We have also calculated the final collision energy distributions $\left(\mathrm{E}_{\mathrm{col}} \mathrm{I}^{\prime}\right)$ of the trajectories at the peaks of $\theta_{\mathrm{v}}{ }^{\prime}$ distributions, which also show a maximum. Thus, for $\mathrm{T}_{\text {surf }}=150 \mathrm{~K}$ and two collision energies $(0.291$ and $0.614 \mathrm{eV})$ the $\mathrm{E}_{\text {col }}$ peaks are located around 0.24 and $0.36 \mathrm{eV}$, which are quite close to the values estimated by using classical rigid molecular scattering calculations ${ }^{4}$ at the same conditions (i.e., around 0.17 and $0.37 \mathrm{eV}$ ).

\section{B. Hyperthermal initial conditions}

Some simulations were also run at hyperthermal conditions for $\mathrm{T}_{\text {surf }}=503 \mathrm{~K}$ and $\theta_{\mathrm{v}}=45^{\circ}$ in order to compare with the experimental polar scattering angular distributions of $\mathrm{O}_{2}$ over highly oriented graphite $^{5}$. A large number of trajectories (60000) were calculated simulating exactly the experimental hyperthermal $\mathrm{O}_{2}$ velocity distribution, whose average translational energy corresponds to $10.46 \mathrm{eV}$, assuming that at these conditions the $\mathrm{O}_{2}$ molecule is at $\mathrm{v}=0$ and $\mathrm{j}=1$ vibrorotional state. 
The calculated distribution is shown in Figure 6. It presents a maximum centred at $\theta_{\mathrm{v}}{ }^{\prime}=73^{\circ}$, a bit larger than the experimental one ${ }^{5}$, which is peaked at $\theta_{\mathrm{v}}{ }^{\prime}=63^{\circ}$. However, the width of the distribution is in good agreement with the experimental one. Since the experimental initial hyperthermal flux consisted of a mixture of both $\mathrm{O}_{2}$ and $\mathrm{O}$ species (with a $\mathrm{O}_{2} / \mathrm{O}$ ratio of 0.48 and with an average translational energy for atomic oxygen of $5.2 \mathrm{eV}$ and for molecular oxygen of $10.46 \mathrm{eV}$ ) these distributions should not directly compared without taking into account some observations. In previous works ${ }^{9,10}$ we reported the scattering distribution of the atomic $\mathrm{O}$ impinging a clean surface and an Oprecovered surface. In the case of a hyperthermal $\mathrm{O}$ beam colliding with a clean graphite surface, the QCT ${ }^{9}$ and the experimental ${ }^{5}$ distributions practically matched the maximum position $\left(\theta_{\mathrm{v}}{ }^{\prime}=65^{\circ}\right.$ and $62^{\circ}$, respectively) and also the distribution shape. Moreover, $\mathrm{O}_{2}$ molecules formed by Eley-Rideal reaction were also calculated by using QCT at the same hyperthermal atomic conditions ${ }^{10}$. These EleyRideal formed $\mathrm{O}_{2}$ molecules could contribute to the final experimental observed $\mathrm{O}_{2}$ polar scattering angular distribution together with the reflected $\mathrm{O}_{2}$ molecules. This possible contribution is also plotted in Figure 6, which is peaked at much lower angles $\left(\theta_{v}^{\prime}=37^{\circ}\right)$. However, in spite of the Eley-Rideal probability is high (i.e., 0.489) when an oxygen atom is preadsorbed over the unit cell at these hyperthermal conditions ${ }^{9}$, the necessary previous $\mathrm{O}$ adsorption step over a clean surface has a low probability (i.e., 0.0016). Therefore, the intensity of the Eley-Rideal contribution to the total polar scattering angular distribution should be in principle very small compared with that originated only by the scattered $\mathrm{O}_{2}$ molecules from the initial beam. This simple analysis assumes a previous clean and pristine graphite surface. However, in the experiments the steady-state oxygen coverage seems to be very high. Highly oriented graphite surface exposed to a flux of hyperthermal oxygen atoms becomes functionalized with epoxides to a concentration between 1 or 2 oxygen atoms per 6 surface $\mathrm{C}$ atoms, as some direct dynamics calculations also support ${ }^{5}$. The high final flux intensity of $\mathrm{O}_{2} / \mathrm{O}$ ratio observed in 
the experiments respect to the initial low value of 0.48 is interpreted as the Eley-Rideal mechanism should contribute highly to the final $\mathrm{O}_{2}$ measured distribution. In such case, the addition of this EleyRideal contribution to the final QCT total polar scattering angular distribution would produce a curve peaked at lower angles and even much closer to the experimental one.

\section{Summary and conclusions}

The study of the collisions of molecular oxygen over graphite surface has been carried out over our recent published analytical flexible periodic LEPS potential energy surface based on DFT data. This surface was previously used for studying the atomic oxygen collisions over a clean and an Opreadsorbed graphite surface with good results in comparison with experimental data.

Only $\mathrm{O}_{2}$ reflection is observed even at high collision energies and vibrational excitation, in agreement with the low expected molecular dissociation for a free of defects surface, supported by previous studies ${ }^{2}$.

Polar scattering angular distributions of molecular oxygen are in agreement with experimental results at several collision energies and surface temperatures. The reflected molecules lose mainly collision energy, which is primarily transferred to the surface. This energy is mostly lost from the perpendicular component of the collision energy, being the parallel one quite conserved, in reasonable agreement with the usual hard cube model assumption. The vibrational level is maintained during the collision and there is a high rotational excitation in all conditions.

The short average collision times along with the analysis of several plots support a single collision dynamics mechanism as was concluded in earlier theoretical studies.

The study at hyperthermal conditions shows a good agreement with the experimental polar 
scattering angular distributions, which would be even better with the inclusion of the calculated $\mathrm{O}_{2}$ Eley-Rideal contribution to the final total distribution.

The very good agreement obtained with the two different experimental studies for a wide range of conditions allows to conclude that the present FPLEPS PES is accurate enough to provide good results on the dynamics for several processes involving atomic and molecular oxygen over graphite surface (i.e., $\mathrm{O}+$ graphite, $\mathrm{O}_{2}+$ graphite or $\mathrm{O}+\mathrm{O}$-graphite) although the inclusion of some defects and a large oxygen coverage on the graphite surface would be possibly necessary to obtain better results.

\section{Acknowledgements}

This work was supported in part by the Spanish Ministry of Science and Innovation (Project CTQ2009-07647), by the Autonomous Government of Catalonia (Project 2009SGR1041) and by the European Commission research funding (Project FP7-SPACE-2009-242311). We thank to Dr. Jianming Zhang for providing us with the experimental initial velocity and translational energy distributions of atomic and molecular oxygen in the experiments for hyperthermal $\mathrm{O}\left({ }^{3} \mathrm{P}\right) / \mathrm{O}_{2}$ colliding over highly oriented graphite and also to Dr. Takahiro Kondo for fruitful comments on the experimental conditions. 


\section{TABLES}

Table I. Fwhm and maximum peak value of the polar scattering angular distributions along with the estimated collision energy loss of oxygen molecules for several initial collision energies and surface temperatures.

\begin{tabular}{c|c|cc|cc|cc}
\hline \multirow{2}{*}{$\mathrm{E}_{\mathrm{col}} / \mathrm{eV}$} & \multirow{2}{*}{$\mathrm{T}_{\text {surf }} / \mathrm{K}$} & \multicolumn{2}{|c|}{$\mathrm{fwhm}^{\circ}$} & \multicolumn{2}{c|}{$\theta_{\mathrm{v}}{ }^{\circ}(\max ) /^{\circ}$} & \multicolumn{2}{c}{$\mathrm{E}_{\text {col loss } / \%{ }^{\mathrm{b}}}$} \\
\cline { 3 - 7 } & & Exp. $^{3}$ & $Q C T$ & Exp. $^{3}$ & $Q C T$ & $H C M^{3}$ & $Q C T$ \\
\hline \multirow{3}{*}{0.291} & 150 & 12.0 & 11.9 & 52.4 & 47.8 & 41 & 35 \\
& 200 & $12.9^{\mathrm{a}}$ & 12.2 & 51.9 & 46.9 & 39 & 34 \\
& 250 & $13.8^{\mathrm{a}}$ & 12.5 & 51.6 & 46.7 & 37 & 33 \\
& 300 & $14.4^{\mathrm{a}}$ & 12.8 & 51.4 & 46.7 & 36 & 32 \\
& 400 & $15.5^{\mathrm{a}}$ & 13.0 & 50.8 & 46.6 & 34 & 30 \\
\multirow{2}{*}{0.371} & 500 & 16.4 & 14.3 & 50.0 & 46.5 & 30 & 28 \\
\hline \multirow{2}{*}{0.456} & 150 & 10.6 & 12.1 & 52.3 & 48.5 & 40 & 37 \\
& 500 & 14.8 & 14.3 & 50.4 & 47.0 & 32 & 32 \\
\hline \multirow{2}{*}{0.534} & 150 & 9.8 & 11.8 & 52.3 & 49.2 & 40 & 40 \\
& 500 & 13.6 & 14.3 & 51.1 & 47.9 & 35 & 35 \\
\hline \multirow{2}{*}{0.614} & 150 & 9.1 & 11.6 & 52.4 & 49.9 & 41 & 42 \\
& 500 & 12.4 & 13.4 & 51.3 & 48.4 & 36 & 38 \\
\hline
\end{tabular}

${ }^{a}$ data obtained from Figures $2 b$ and $2 c$ of Ref. 3 .

${ }^{b}$ the percentage of energy loss is calculated by $\left(1-\frac{E_{c o l}{ }^{\prime}}{E_{c o l}}\right) \times 100$, where $E_{c o l}$ and $E_{c o l}$ stand for initial (incident) and final (scattered) collision energies, respectively. 


\section{FIGURE CAPTIONS}

Figure 1. Coordinate system for $\mathrm{O}_{2}$ molecule interacting with a graphite (0001) surface. The internal coordinates $(\mathrm{r}, \theta, \phi)$ define the molecular oxygen orientation while $\left(\theta_{\mathrm{v}}, \phi_{\mathrm{v}}\right)$ define the angle of incoming centre of mass velocity vector $\left(\overrightarrow{\mathrm{v}}_{\mathrm{cm}}\right)$.

Figure 2 DFT (upper panels) and FPLEPS (bottom panels) equipotential energy contour plots for two $\phi$ angles $\left(\phi=0^{\circ}\right.$ and $60^{\circ}$, left and right panels, respectively) for parallel approach $\left(\theta=90^{\circ}\right)$ of incoming $\mathrm{O}_{2}$ with a perpendicular velocity to the surface $\left(\theta_{\mathrm{v}}=0^{\circ}\right)$. The contour plots are depicted in increments of $0.5 \mathrm{eV}$, and the zero of energy is taken as $\left[\mathrm{O}_{(\mathrm{g})}+\mathrm{O}_{(\mathrm{g})}\right]$. The value of $0 \mathrm{eV}$ of energy is labelled and corresponds to the nearest solid line.

Figure 3 QCT polar scattering angular distributions $\left(\theta_{\mathrm{v}}{ }^{\prime}\right)$ of the reflected $\mathrm{O}_{2}$ molecules for $\theta_{\mathrm{v}}=0^{\circ}$ (left panels) and $45^{\circ}$ (right panels) without thermal bath ( $\left.a, b\right)$ and considering two surface temperatures $\left(T_{\text {surf }}=100 \mathrm{~K}(\mathrm{c}, \mathrm{d})\right.$ and $\left.900 \mathrm{~K}(\mathrm{e}, \mathrm{f})\right)$ for several initial collision energies $\left(\mathrm{E}_{\mathrm{col}}=0.2,0.5\right.$ and $1.2 \mathrm{eV}$ using solid, dashed and dotted lines, respectively). The impinging $\mathrm{O}_{2}$ molecules were in $\mathrm{v}=0$ and $\mathrm{j}=1$ vibrorotational states. The distributions are normalized to unit area.

Figure 4 Transfer energy analysis (collision, internal and total energy in the upper, medium and bottom panels, respectively) at two surface temperatures: $\mathrm{T}_{\text {surf }}=100 \mathrm{~K}$ (left panels) and $900 \mathrm{~K}$ (right panels) for several initial collision energies: $\mathrm{E}_{\mathrm{col}}=0.2$ (solid line), 0.5 (dashed line) and 1.2 (dotted line) $\mathrm{eV}$ and normal incidence. The values in parentheses correspond to the maximum of the distribution for each value of initial collision energy. 
Figure 5 Initial collision energy $\left(T_{\text {surf }}=150 \mathrm{~K}\right.$, left panels $)$ and surface temperature $\left(E_{\text {col }}=0.291 \mathrm{eV}\right.$, right panels) dependency of the QCT polar scattering angular distributions $\left(\theta_{v}^{\prime}\right)$ of the reflected $\mathrm{O}_{2}$ molecules scattered from the graphite surface (solid circles) and the comparison with the experimental data ${ }^{3,4}$ (solid lines) for $\theta_{\mathrm{v}}+\theta_{\mathrm{v}}{ }^{\prime}=90^{\circ}$ restriction. Right axes correspond to right panels. The QCT distributions are normalized at each corresponding experimental peak.

Figure 6 QCT Polar scattering angular distributions $\left(\theta_{\mathrm{v}}^{\prime}\right)$ of $\mathrm{O}_{2}$ for initial experimental hyperthermal conditions $\left(<\mathrm{E}_{\text {col }}>=10.46 \mathrm{eV}\right)$ with $(\mathrm{v}=0, \mathrm{j}=1), \theta_{\mathrm{v}}=45^{\circ}$ and $\mathrm{T}_{\text {surf }}=503 \mathrm{~K}$. Reflected $\mathrm{O}_{2}$ molecules (solid line), $\mathrm{O}_{2}$ molecules formed via Eley-Rideal reaction on an O-precovered surface ${ }^{10}: \mathrm{O}_{(\mathrm{g})}+\mathrm{O}_{(\mathrm{ad})}$ $\rightarrow \mathrm{O}_{2(\mathrm{~g})}$ (dashed line) and the experimental data (dotted line ${ }^{5}$ ). All curves are normalized to unit at the maximum. 
Figure 1

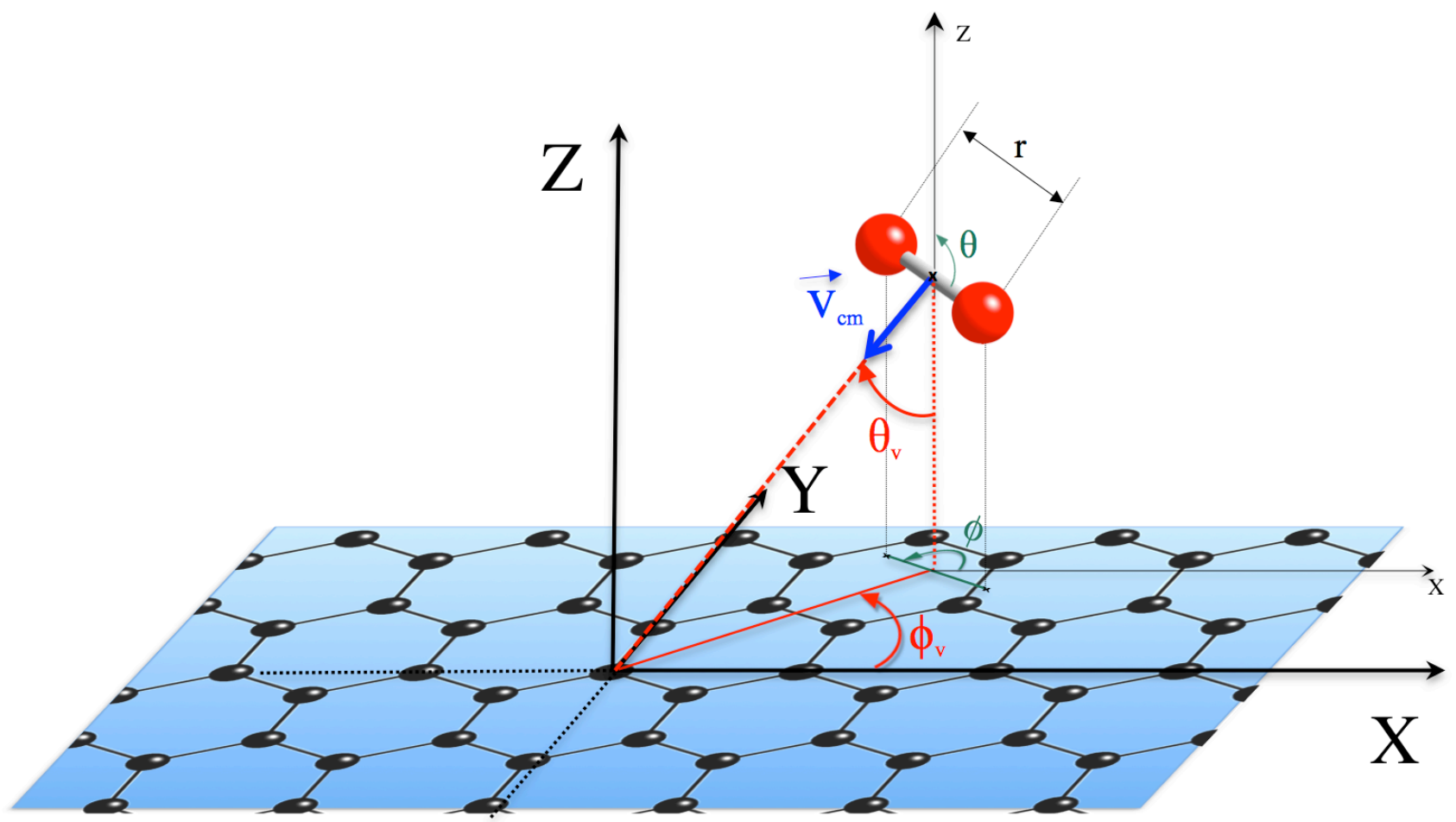


Figure 2

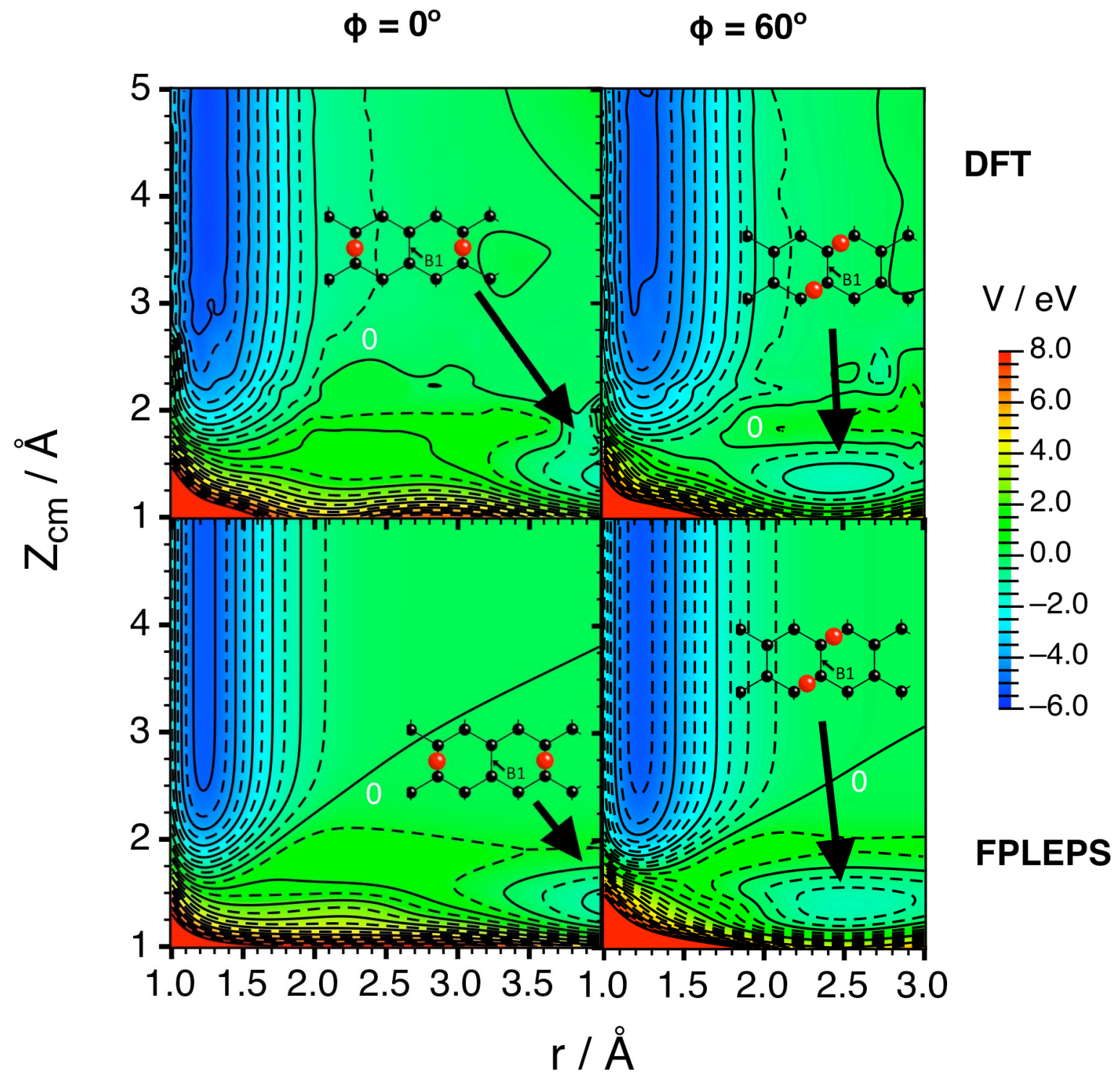


Figure 3

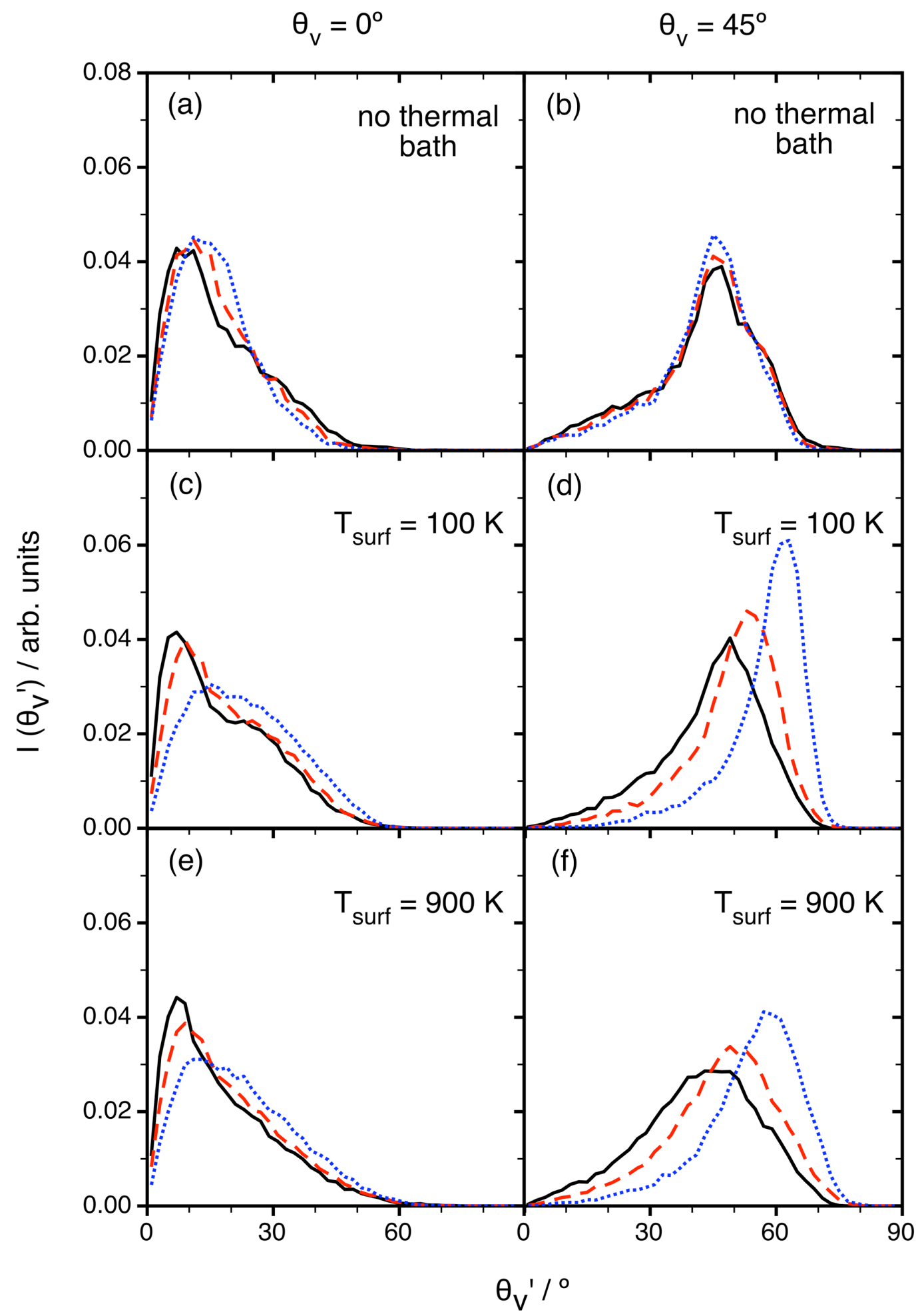


Figure 4

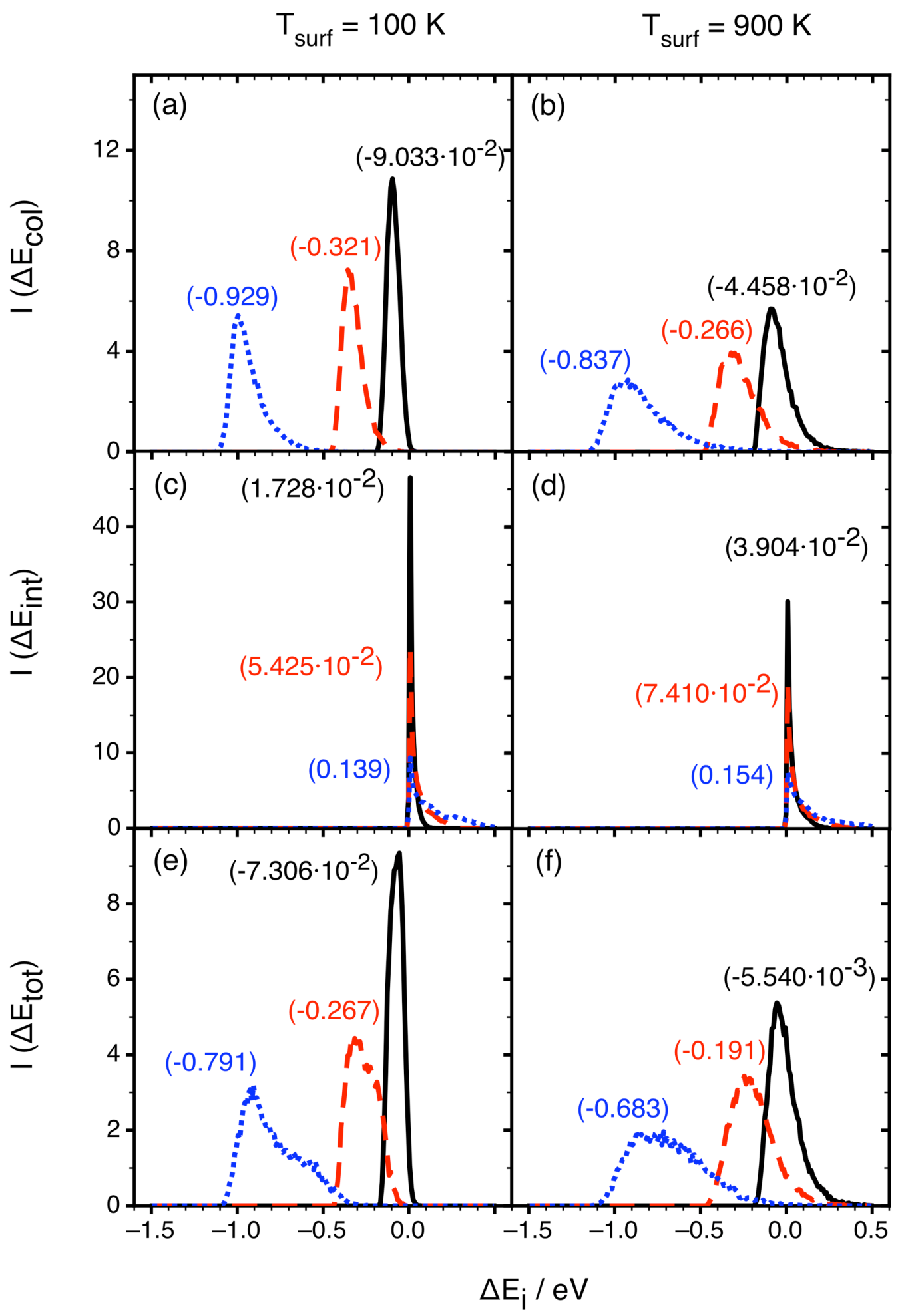


Figure 5

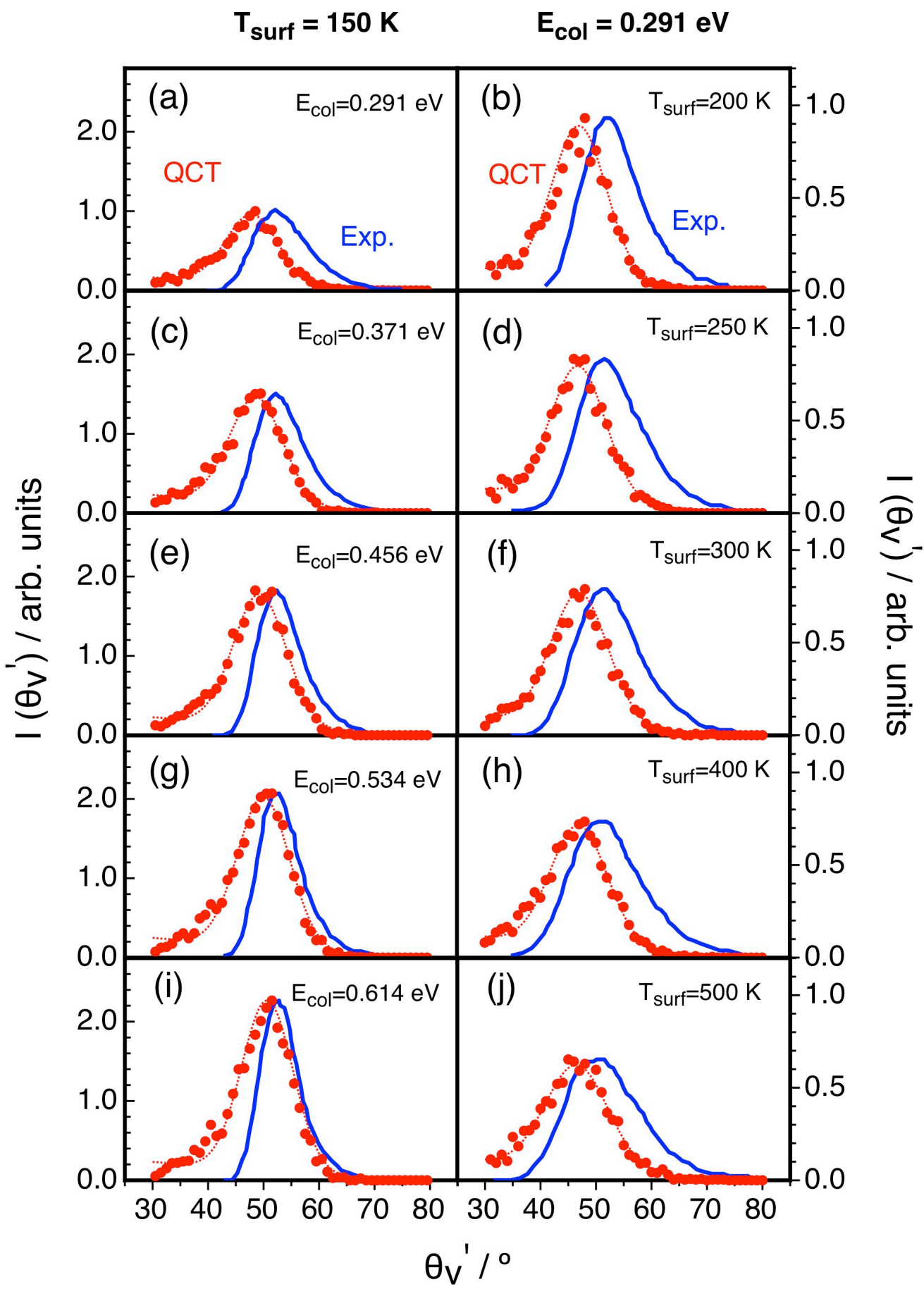


Figure 6

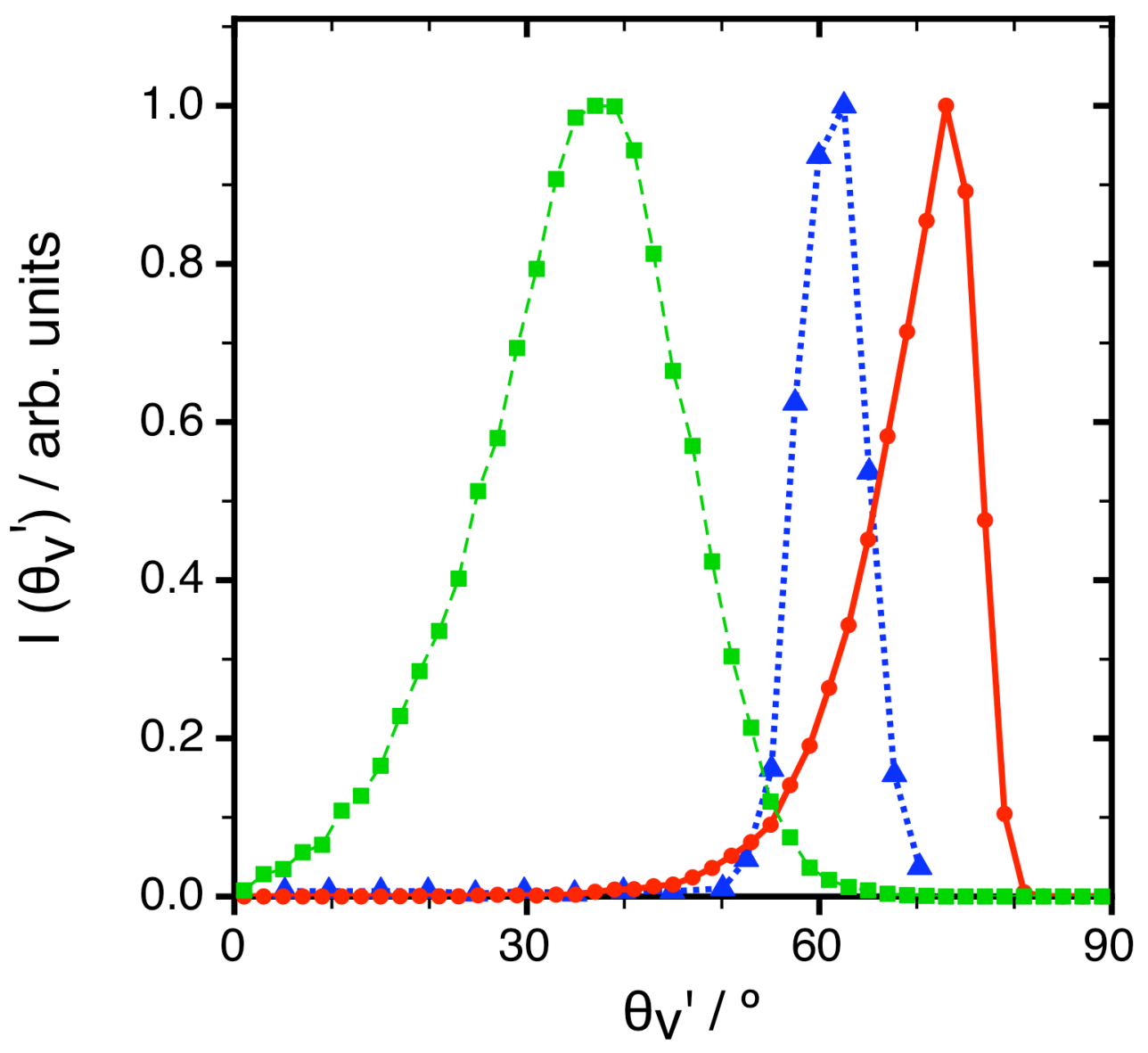




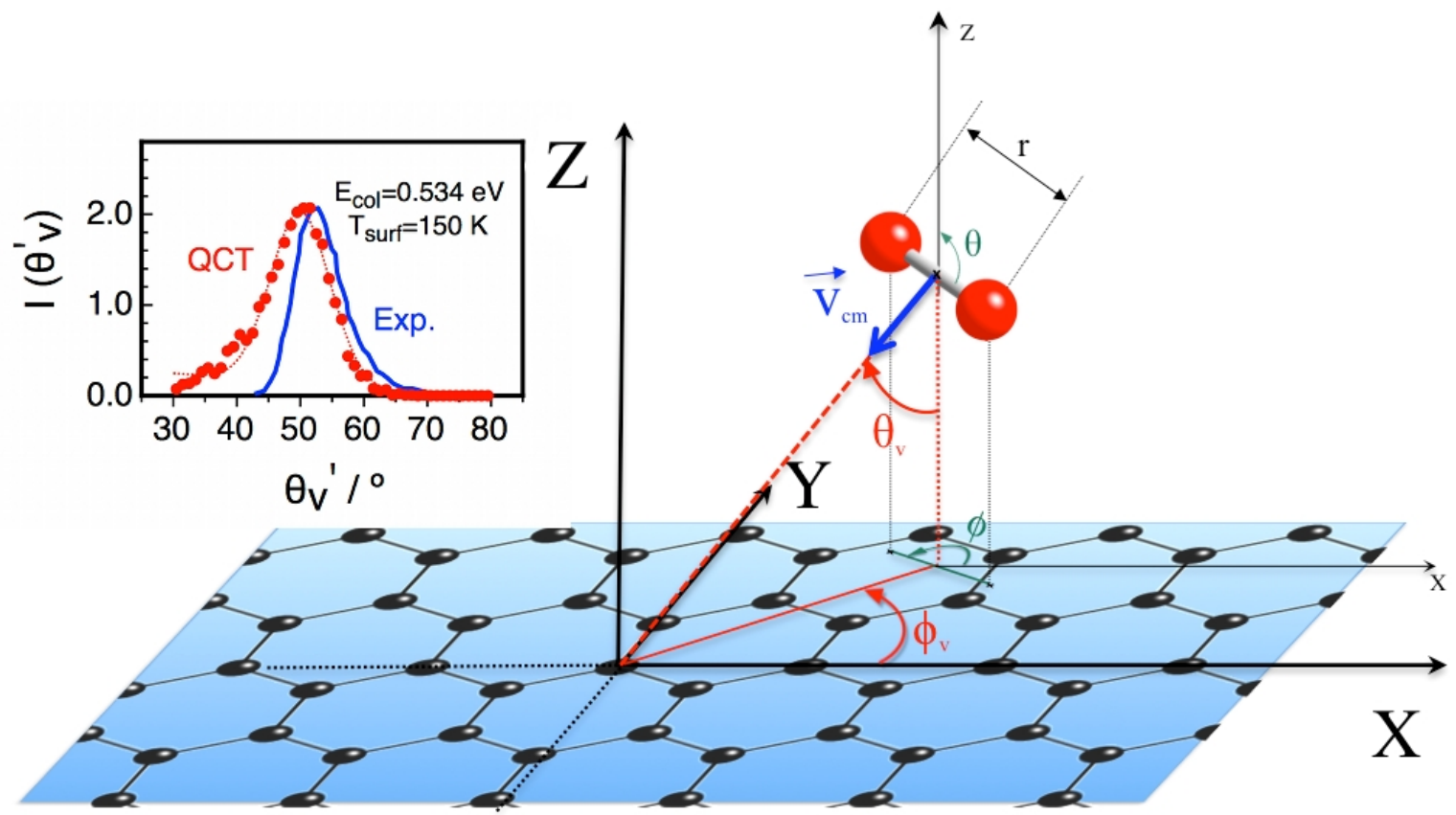




\section{References}

1. Ngo, T.; Snyder, E. J.; Tong, W. M.; Williams, R. S.; Anderson, M. S. Surf. Sci. Lett. 1994, 314, L817-L822.

2. Xu, S. S.; Chen, H-L.; Lin, M. C. J. Phys. Chem. C 2012, 116, 1841-1849.

3. Oh, J.; Kondo, T.; Arakawa, K.; Saito, Y.; Hayes, W. W.; Manson, J. R.; Nakamura, J. J. Phys. Chem. A 2011, 115, 7089-7095.

4. Hayes, W. W.; Oh. J.; Kondo, T.; Arakawa, K.; Saito, Y.; Nakamura, J.; Manson, J. R. J. Phys.: Condens. Matter 2012, 24, 104010:1-9.

5. Paci, J. T.; Upadhyaya, H. P.; Zhang, J.; Schatz, G. C.; Minton, T. K. J. Phys. Chem. A 2009, 113, 4677-4685.

6. Nicholson, K. T.; Minton, T. K.; Sibener, S. J. J. Phys. Chem. B 2005, 109, 8476-8480.

7. Kinoshita, H.; Umeno, M.; Tagawa, M.; Ohmae, N. Surf. Sci. 1999, 440, 49-59.

8. Morón, V.; Gamallo, P.; Sayós, R. Theor. Chem. Acc. 2011, 128, 683-694.

9. Morón, V.; Martin-Gondre, L.; Crespos, C.; Larregaray, P.; Gamallo, P.; Sayós, R. Comp. Theor. Chem., 2012, 990, 132-143.

10. Morón, V.; Martin-Gondre, L.; Gamallo, P.; Sayós, R. J. Phys. Chem. C 2012, 116, 13092-13103.

11. Kresse, G.; Hafner, J. Phys. Rev. B 1993, 47, 558-561.

12. Kresse, G.; Hafner, J. Phys. Rev. B 1994, 49, 14251-14269.

13. Kresse, G.; Furthmüller, J. Comput. Mater. Sci. 1996, 6, 15-50.

14. Kresse, G.; Furthmüller, J. Phys. Rev. B 1996, 54, 11169-11186.

15. Billing, G. D. Dynamics of Molecule Surface Interactions, John Wiley \& Sons: New York, 2000 (Chap. 5 and 6).

16. Kondo, T. private communication.

17. Huber, K.; Herzberg, G. Constants of diatomic molecules (Van Nostrand Reinhold Company, New York, 1979).

18. Tully, J. C. J. Chem. Phys. 1980, 73, 1975-1985.

19. Polanyi, J. C.; Wolf, R. J. J. Chem. Phys. 1985, 82, 1555-1566.

20. Busnengo, H. F.; Di Cesare, M. A.; Dong, V.; Salin, A. Phys. Rev. B 2005, 72, 125411-125419.

21. Groenenboom, G.; Struniewicz, I. J. Chem. Phys., 2000, 113, 9562-9566.

22. Barker, J. A.; Auerbach, D. J. Faraday Discuss. 1985, 80, 277. 